\title{
Vulnerability and industrial hazards in industrializing countries: an integrative approach
}

\author{
M.F. de Souza Porto *, C.M. de Freitas \\ Study Center of Workers' Health and Human Ecology, National School of Public Health, Oswaldo \\ Cruz Foundation, Av. Leopoldo Bulhões, 1480 Manguinhos, 21041-210 Rio de Janeiro, Brazil
}

\begin{abstract}
The extra vulnerability of industrializing countries to environmental problems and industrial accidents cannot be understood or solved by a 'normal' scientific analysis. Aspects of the social and institutional context must be included, through analyses based on post-normal science. The standard two-dimensional classification of PNS is modified to have axes 'social 'and institutional vulnerabilities' and 'complexity of technological hazards'. The analysis is mainly applied to the case of the relatively rare accidents with catastrophic potential. In these, the deaths per accident in India, Mexico and Brazil are much greater than in the industrialized countries. This discrepancy arises partly from location of such plants near residential communities for marginalized workers and their families. Other socio-political factors are relevant, as the role of these countries in the global production system, the enforcement of safety and planning laws, quality of housing, and lifestyle of residents. Reducing the vulnerability of industrializing countries will therefore require major social policies and a comprehension of the limits of the normal scientific and economic approaches to such problems.
\end{abstract}

(C) 2003 Elsevier Science Ltd. All rights reserved.

\section{Introduction}

The scientific analysis and the proposed solutions to environmental problems can be seriously limited by methodological and epistemological factors. Understanding this is very important if we want to resolve the difficulties in solving some specific environmental problems in the context of industrializing countries such as Brazil.

* Corresponding author. Fax: +55-21-270-3219.

E-mail address: firpo@ensp.fiocruz.br (M.F. de Souza Porto). 
The point has special relevance to those problems which in industrialised countries have technical solutions and are more or less efficiently managed.

The aim of this essay is to examine the vulnerability of the so-called industrializing countries regarding some environmental risks. It is based on our experience in developing interdisciplinary studies to understand industrial hazards in Brazil, mainly major chemical accidents. The conceptual references for this article are taken from the discussions about vulnerability [1,2], interdisciplinary studies and complex systems [3], and specially the contributions of Post-Normal Science [4-6] approach.

We put forward the hypothesis that present socio-political, socio-economic and institutional factors in industrializing countries make them much more vulnerable to some environmental problems and create the type of settings where technical approaches used successfully in highly industrialized and institutionalized societies prove less effective when analyzing and proposing solutions in the context of countries like Brazil.

We argue that the development of socio-technical approaches is very important for the understanding and solution of environmental problems, or at least for the understanding of the enormous difficulties and limits involved. As a consequence, the role of institutions and professionals can be enhanced by the awareness that we cannot solve the whole problem in the short term, but we can incorporate questions and methods capable of improving social and institutional dynamics related to structural changes of society in order to promote a better quality of life.

Although the Post-Normal Science approach has been developed in the context of high industrialized societies, we understand that the focus on science, quality and "the extended peer communities" can illuminate the application of the systems approach to issues of vulnerability in reference to industrial risks in industrializing countries. For this, we discuss the relation between the PNS diagram-which links the systems uncertainties and decision stakes in three levels of problem-solving strategies - and another diagram which comprehends complex socio-technical systems in industrializing countries as a result of vulnerability and technological hazards. In other words, more simple technological hazards can become complex socio-technical systems if we analyze them taking into consideration the vulnerabilities of a society. In this sense, the assumptions of PNS can be applied even for more simple systems where uncertainties are not directly the result of intrinsic technological risks, but expressions of vulnerabilities, which are inseparable from explicit considerations of ethics and policy. Here the concept of "Extend Peer Community" should specially include the most vulnerable social groups and strategies of empowerment in order to transform them in real stakeholders.

\section{Environmental problems, interdisciplinarity and the Post-Normal Science approach}

There are two groups of limitations related to the way we face environmental and technological hazards. The first type has an epistemological and methodological basis and refers to the formulation of modern normal sciences. The second is concerned 
with social and institutional aspects of societies that can increase the vulnerability of specific populations and regions facing environmental problems. To deal with these questions we make use of the contributions of Post-Normal Science (PNS) and interdisciplinary approaches, and the work of some authors who discuss the concept of vulnerability.

According to Funtowicz and Ravetz [4-6], a new kind of science is emerging in response to the challenges of policy issues regarding environmental and other risks. The theory of Post-Normal Science is formulated in contrast both with the concept of Kuhn [7] of normal science and the notion of post-modern, a term for describing contemporary cultural phenomena associated with nihilism and despair. Kuhn related his concept to the routine of puzzle solving by which science progresses steadily during the periods between conceptual revolutions. Here uncertainties in knowledge are managed automatically in a "neutral" position where values are not explicit. In terms of systems theory, this means a reduction of complexity of the natural and social worlds to the mere complication or even simplicity of an industrialized "mechanical world", symbolized by mathematical representations applied to scientific prediction and mechanical control.

Undoubtedly, this model of normal science contributed great advances and comfort to modern civilization. But this conception is no longer appropriate in the new context of environmental problems with large temporal and spatial scales, along with strong ethical aspects and elements of uncertainty. The dominant scientific method based on the study of an isolated piece of nature that is kept unnaturally pure, stable and reproducible is being reviewed due to the global environmental risks and their associated threats for the future.

In response to the new challenges, PNS has been developed as a problem-solving strategy applied when facts are uncertain, values in dispute, stakes high and decisions urgent, in a context of a need for effective quality assurance. It also includes legitimating and extending participation in the evaluation process in what Funtowicz and Ravetz [5] called an "Extended Peer Community", "including all stake-holders in an issue who are prepared for a dialogue", regardless of their formal certification, improving the quality of the decision process.

Some industrial risks can become complex if we analyze them within a sociotechnical system, based on systemic and interdisciplinary approaches. According to Garcia [3], interdisciplinarity is a methodological answer to complex problems for which isolated normal disciplinary analysis would not give good results, since the analyzed reality has multiple and interrelated dimensions.

The interdisciplinary approach looks for the successive generation of integrated synthesis during the study through systemic interpretations and integrative diagnosis, enabling results not obtainable by isolated specialist analysis. For Garcia, an interdisciplinary study is problem oriented. It begins with the initial formulation of the problem, demarcating a common conceptual framework joining the involved specialists in the construction of the main elements and relations characteristic of a complex system. It also means that a single initial problem can have multiple interdisciplinary approaches depending on the interests, priorities and values of the involved insti- 
tutions and researchers, who can conceive distinct sets of hierarchies and relationships within the analyzed system.

The evolution of an interdisciplinary study follows an interactive and non-linear process in the medium term through a dialectic movement between the differentiating (application of specialized methods and analysis) and integrative (integration of results in comprehending and proposal of solutions) phases, and between the diagnosis and the generation of alternative proposal stages.

As suggested by the PNS approach the capability of dialogue within the research team and the involved social actors and institutions are essential for the success of an interdisciplinary study. The possibility of this interface dialogue between different professionals as well as paradigms depends on an ethical and intellectual basis, where values are explicit and uncertainties are assumed as conditions to recognize the participation of the "extended peer community". Here, the concept of complexity also means a plurality of perspectives that cannot be reduced to each other.

The PNS and interdisciplinary approaches are examples of a new tendency in the practices of science and institutions, which searches for answers to the crisis of the classical scientific knowledge and to the increasing complexity of modern civilization, especially concerning environmental problems. There is a consensus that the decisions based on scientific certainty are no longer sufficient to guarantee democracy and quality of life for the future generations. The precautionary principle and sustainable development call for efforts to develop new paradigms, which is in correspondence with the theory of Post-normal Science in solving complex environmental problems, including vulnerable areas.

\section{Vulnerability and environmental problems in industrialising countries}

The systems theory, the concept of complexity and the PNS approach are very important contributions for comprehending environmental problems and generating alternative proposals, especially concerning complex problems such as global environmental risks. With its conceptual and methodological support, we developed a model for a better understanding of environmental problems in industrializing countries, where existing social, institutional and economic structures make these sociotechnical systems much more vulnerable to such problems through the aggravation of risk situations and events.

From our point of view [8], the comparative inability of industrializing countries to control industrial risks is linked to the contemporary global economy as a system characterized by the interdependence between countries, where the production of goods for the world market is not merely the main goal, but also requires the development of financial and technological exchanges $[9,10]$. In this interdependent system, each and every country has its role in the international division of labor, and this leads to an international sharing of benefits and also risks. More than $80 \%$ of the global consumption of goods is restricted to a quarter of the world's population, mostly living in industrialized countries [11]. The positions are inverted when we examine risks. In industrializing countries, a reduction in the number of official meas- 
ures for the protection of the environment, human health and safety have been considered, although not always explicitly, an important item in global economic negotiations and these have often led to an unfair international division of risks [12]. The recent financial crisis related to the globalization process and the increasing capital mobility in the so-called emergent countries in Asia and Latin America represents another aspect of their vulnerability. The concept of vulnerability has its significant origin in the studies of "man-made" and "natural" disasters. It tries to explain how similar hazards can produce different effects in distinct countries and populations, introducing the relevance of socio-economic factors and economic development in generating susceptibility to the effects of natural and man-made hazards [1,2]. Horlick-Jones [1] defines vulnerability as a unifying concept based on the systems theory, as erosion of a system's resilience to perturbations generated by the interaction of a complex socio-technical system vulnerable to failure with a vulnerable population within a socio-economic environment. Thus, vulnerability becomes a transdisciplinary concept integrating different specialists and methods in mapping risk situations in geographical and social spaces.

The concept of vulnerability provides the basis for an integrated and transdisciplinary approach, where social scientists, economists, geographers, engineers, toxicologists among others can work together, combining qualitative and quantitative methods in order to interpret the socio-technical system and develop alternative proposals that could not be generated by isolated methods taken from normal sciences.

The concept of vulnerability in industrializing countries regarding industrial risks can also be associated with other references. The first reference is the concept of coupling for studying mixed technological and societal systems with the different sorts of uncertainty in the different phases of risk assessment and management [1315]. The second arises from the discussions about the different decision-making processes in different societies involving the acceptability and regulation of technological risks [16,17]. The international division of hazards and the double standards between industrialized and industrializing countries [18] and the discussions on environmental justice [19] are also important references related to social vulnerability.

\section{Vulnerability and the socio-technical approach in industrialising countries}

Industrial hazards in developing countries are good examples to understand the effectiveness of the concept of vulnerability in an integrative approach. They can be interpreted within a social-technical system, where socio-politic, socio-economic, institutional and technological factors are strongly related and can help us understand how present social and political structures in industrializing countries have contributed to the aggravation of risk situations and events. Fragmented studies on the Risk Analysis field cannot determine what kind of structural elements are central to conditioning and solving environmental risks. As pointed out by Kasperson et al. [20] "the practice of characterizing risk by its probability and magnitude of harm has drawn fire for neglecting equity issues in relation to time (future generations), 
space (the so-called LULU or NIMBY issue), or social groups (the proletariat, the highly vulnerable, the export of hazard to developing countries)".

Fig. 1 illustrates the relationship between the global and local levels in what we can call a socio-technical analysis of industrial hazards. At the bottom of Fig. 1risk events with damages - we can analyze different stages in the production of risk

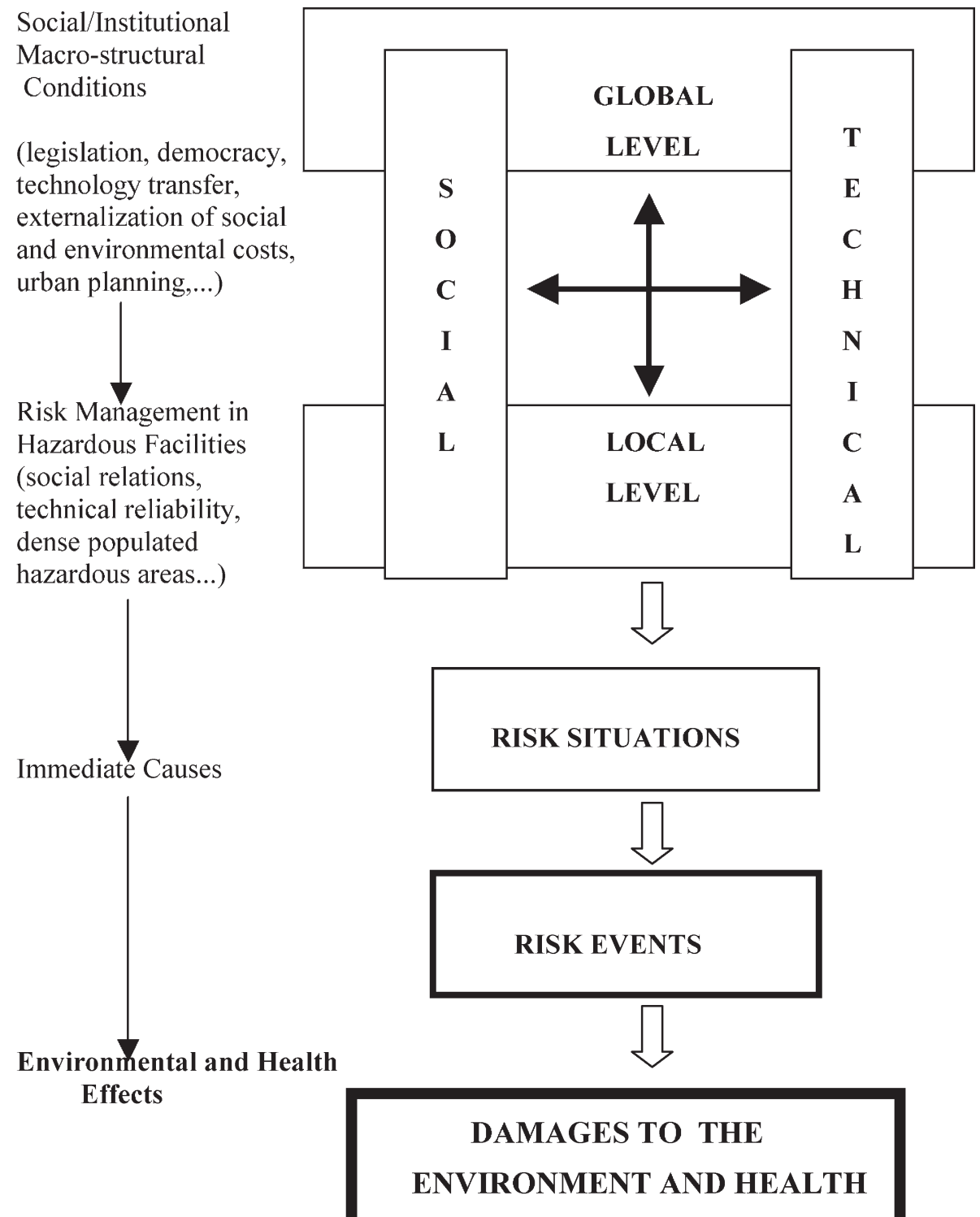

Fig. 1. Socio-technical system and technological hazards. 
situations in a local level-for example, facilities in specific areas. Immediate technical causes of accidents are preceded by underlying factors within the companies, related to managerial, organizational and procedural aspects, as well as design inadequacy. But these structural causes present in a single or in groups of companies are also expressions of social and institutional macro-structural conditions of a society. They influence the risk management policies and the consequences of risk events in specific facilities, groups and regions. As examples of these structural conditions, we can mention legislation, democracy within the decision processes and social relations of labor, technology transfer, externalization of occupational and environmental costs and urban planning.

The interaction between these structural elements to risk situations and events has a non-linear route, through social, economic and institutional processes coupled with technological characteristics of a production system. In this way, the socio-technical analysis of accidents configures a hierarchic relationship between multiple elements, which are focused depending on specific regions, economic sectors and technologies [21]. Thus, the integrative analysis will try to harmonise different disciplines, concepts and methods around a socio-technical system structured in the relations between, for example, social, institutional, technological, epidemiological and environmental aspects.

In general terms, the social components can be apprehended, among other ways, through the analysis of the economic situation of a country, region or economic sector, existing legal and institution landmarks, and the level of labour organisation and the awareness of the workers. Such elements are as important a part of the analysis of the productive process as the analysis of technical features, involving specialised professionals and operators. This combination of the analysis of macrostructural elements of underlying causes (including management and organisational aspects) with the analyses of concrete risk situations must constitute the evaluations of risk and the technology used in one determined productive process.

Obviously, a failure-a decision or action carried through inadequately or not implemented inside the organisation — can be considered within the macro-structural and technological conditions that make it possible. For example, a machine or a facility can fail to comply not only with the international standards that express the most advanced state of the art in terms of safety in a productive process, but also with the national norms, frequently incomplete or limited. It can also fail to perform the necessary preventive maintenance because of an international crisis in the financial market, where a retraction of the world market forces the most vulnerable companies to cut the necessary safety investments. In Brazil, there are several vulnerable sectors, mainly in small and medium companies but sometimes in big ones, where we can find obsolete equipment and processes. In these sectors, the economic fragility and institutional vulnerability allow the formation of a "culture of improvisation", through inadequate maintenance and hazardous activities, where abnormalities are considered normalities and are incorporated into the organisations, constituting what Wynne [14] calls normal abnormalities. 


\section{Vulnerability and the Post-Normal Science approach: the case of industrial accidents}

Fig. 2 illustrates the relation between vulnerability and technological hazards increasing the complexity of socio-technical systems. This figure is similar to the diagram adopted by Funtowicz and Ravetz [5] to represent the different problemsolving strategies proposed by the PNS theory. But our diagram emphasizes the concept of vulnerability when describing how technically simple hazards can become complex if we analyze them in a socio-technical system, coupling hazards with social and institutional vulnerabilities, which are common in industrializing countries.

The PNS diagram emphasizes the relation between systems uncertainties and decision stakes demanding different problem-solving strategies. According Funtowicz and Ravetz [5], when these two elements "are small, traditional" Applied Science is adequate. But when either is medium in intensity, mere research expertise is insufficient" and may involve personal judgement and responsibility through "Professional Consultancy". Finally, where either uncertainties or stakes are high, a new approach is required and inseparable from explicit considerations of ethics and policy. The PNS is aimed at helping the reorientation of scientific practice to meet these new challenges.

The PNS approach has been developed specially in the context of modern European highly industrialized countries characterized by the relatively well applied social, redistributive and institutional policies, as well as the high level of formally educated citizens and participative decision-making processes. But the reality of industrializing countries such as Brazil is quite different: concentration of wealth, social exclusion, low level of formally educated people, weakness of institutional practices and non participative decision-making processes. Here social inequalities must be emphasised as part of the increased complexity of social-technical systems

\section{Social and Institutional Vulnerabilities}

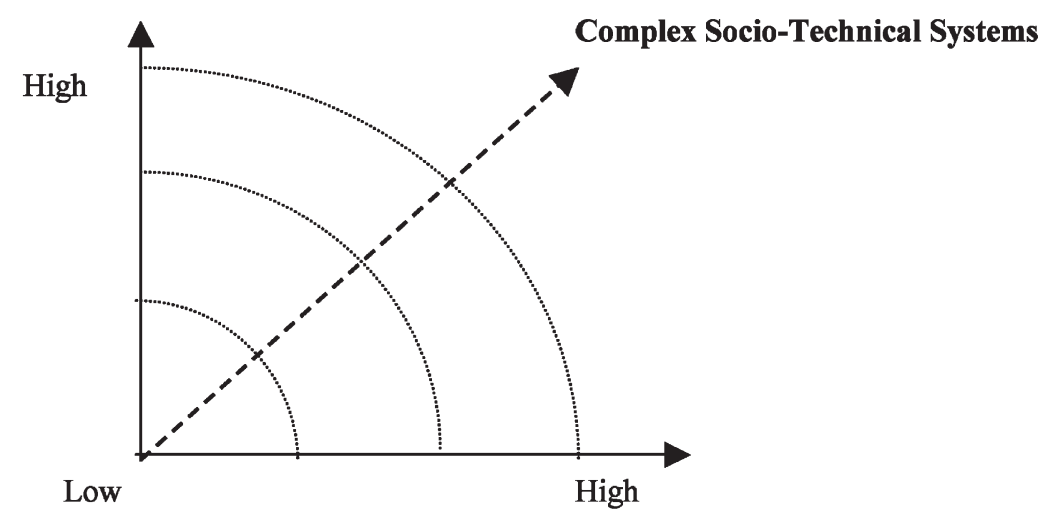

Complexity of Technological Hazards

Fig. 2. Vulnerability, industrial hazards and socio-technical systems. 
which combine traditional and modern social organizations, institutions and technologies, including high risk ones. Abstract decision stakes seem to be artificial in this context if we do not insert them into concrete social groups and institutions with their vulnerabilities. Isolated "Applied Science" or "Professional Consultancy" models, as institutionalized in modern industrialized countries, are not adequate for the analysis and solution of environmental problems in countries where issues of vulnerability are a leading question.

To explain Fig. 2, we will use the examples of three groups of industrial accidents, relating them with social, technological and epidemiological features. The first group involves simple manual workers and accidental falls, typical accidents of civil construction. In social terms, this workforce is characterized by having low technical qualifications and a low level of labour union organization. Technologically, the productive process is associated with the organisation of the construction sites and the work with manual tools, with accidents occurring at high frequency and severity in countries like Brazil. The second type of accident involves the use of machines in specific workplaces, as in the metal processing sector, with very differentiated levels of technical qualification and labor union organizations, normally stronger in big companies, with medium frequency and severity. The third type involves fires, explosions and releases in industries of high technological complexity, such as nuclear, chemical and the petrochemical ones, where tightly linked technical systems generate systemic accidents. These are of low frequency, but with a catastrophic potential in the case of major accidents.

The classic ideas of Safety Engineering are applied mainly to the two first types of accidents. But we can confirm that an isolated technical analysis is not capable of explaining the high incidence of serious accidents in jobs of relatively low technical complexity in civil construction. The non-incorporation of the social dimension in this situation tends to limit the analysis to possible cases of individual recklessness, whereas the aspects of low technical qualification and weak labour organisation of the workers in this area are vital for the understanding of the frequency of easily controllable, although serious accidents. The vulnerability of this category of workers is revealed by the recurrence of falls from trusses. Such simple accidents, together with vulnerable workers and institutions, constitute a complex socio-technical system.

An integrative approach implies the need of widening our conception of the problem, through the integration of social and human sciences with the technological disciplines. Moreover, it becomes imperative to establish institutional strategies for the reduction of vulnerability, through, for example, the empowerment of vulnerable social groups to act as important characters in defining the priorities of the public policies. Risk perception and communication studies need to be reoriented to an integrated assessment where poor and illiterate people can become real participants. The challenge is not easy: how can we break the vicious circle between social exclusion and the decision-making processes, which together define institutional priorities and practices?

On the other hand, the application of the classical notion of safety is especially restrictive in the case of accidents in industries of continuous process. In these indus- 
tries, the work processes normally involve sophisticated technologies, what Perrow [13] calls strongly coupled complex systems. In these systems, dysfunction of certain subsystems can lead to systemic accidents, through the so-called domino effect, where all or a significant part of the system is destroyed, implying damages of enormous value. In these technologies, technical and human reliability needs to be deeply and extensively evaluated and controlled what shows that the classical theory of Safety Engineering is inadequate to the demand. A series of new techniques of risk analysis have been developed, particularly since the 1950s, including the systems tree techniques and the probabilistic calculation of possible failures of components using Boolean mathematical logic. Later, these techniques were spread through industrial sectors of lesser technological complexity, producing a more effective introductory technique for the understanding, analysis and control of industrial accidents.

To comprehend the complexity of socio-technical systems in industrialising countries, the socio-politic and socio-economic components are particularly important, given the social inequalities that determine the vulnerability of diverse categories of workers. For example, accidents type 1 and 2 mentioned above occur in situations of technically unqualified work with little social visibility, low level organisation and little power for the workers. In countries with a generally low dissemination of social welfare policies and precarious conditions of work, there is a rapid turnover of workers, a low level of training of the operatives and a low value attributed to the life and the health of the workers. In general, the work force is considered as capable of inexhaustible substitution. These sectors are particularly resistant to the implementation of safer technologies and processes, and dangerous situations are part of the routine and the culture in the workplace. The mortality rate in these accidents shows a high ratio of male victims, disclosing a perverse culture in the use of male chauvinism and muscular strength. In such a context, it is hardly surprising that there tends to be a perfunctory management of risks. In spite of the common knowledge of this context, accidents still tend to be investigated in terms of individual behaviour, and so are explained in terms of unsafe acts.

The accidents of type 3 are of major interest in this study. At one end of the scale they are notorious, as when they appear as catastrophic explosions, such as the accident of Vila Socó which occurred in Cubatão/Brazil in 1984, with about 500 deaths. But they extend continuously down to the frequent 'releases' which have no acute effects and are therefore not recorded, although their cumulative effect on the neighbouring natural and human environment may be devastating. The productive process in these cases requires operatives with higher qualifications, who may create more powerful organisations. If the workers of the first two groups are stressed by an elevated turnover, these latter are limited in their militancy by the 'privilege' of having a job of better remuneration, as well as by the pressures for reductions on staff promoted by strategies of reengineering and increasing productivity. These latter tendencies naturally increase the risk of the operations. Another aspect, particularly important to the Brazilian reality, is the introduction of less qualified (and less organised) workers into the more hazardous activities. This is accomplished through subcontracting strategies; and it brings the process industries-particularly in the 
activities of maintenance and lesser qualified tasks - into a state of vulnerability similar to that of the two first groups of accidents.

\section{Vulnerability and major chemical accidents in industrialising countries}

Major chemical accidents in industrializing countries are good examples for demonstrating the effectiveness of the socio-technical approach in analyzing vulnerability. Chemical accidents can result from emissions, fires or the explosion of chemicals during transportation, storage or industrial activities, leading to serious, immediate or delayed damage to human beings and/or the environment, and involving one or more chemical substances [22-24]. They are considered major chemical accidents-even if, as argued by Otway et al. [16] the concept of 'major accident' is a rather fuzzy one. Chemical accidents can be analyzed in industrializing countries using the concept of vulnerability within a model where social, technical, institutional and human systems interact with each other, increasing uncertainties and aggravating consequences. Fig. 3 below synthesizes this model of vulnerability, having as reference the model developed by Horlick-Jones [1].

There is literature that indicates a higher rate of fatalities and injuries related to industrial hazards. For example, the worst major chemical accident in the 1980s occurred in industrializing countries-India, Brazil and Mexico-either in multinational corporations (as in Bhopal, India) or in national corporations (as in São

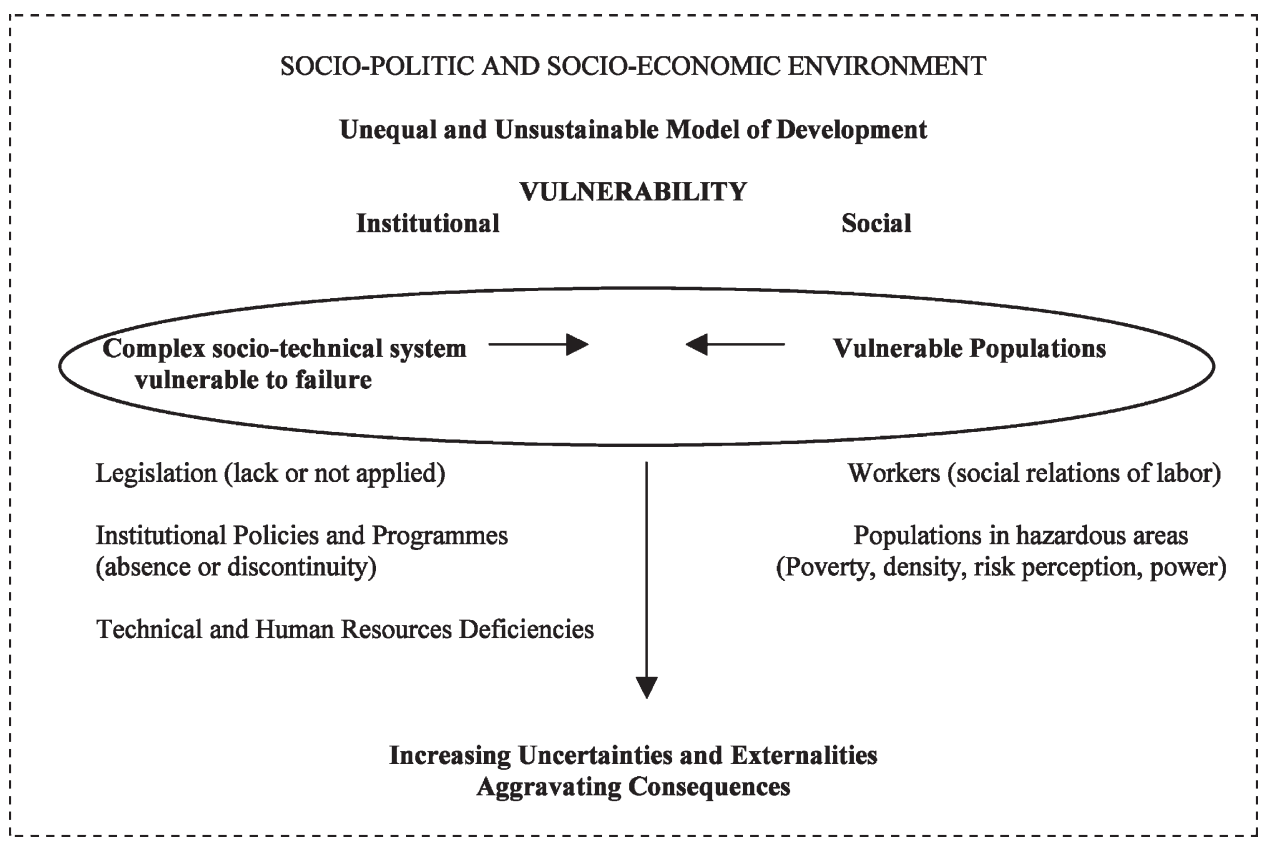

Fig. 3. Model of vulnerability for industrial hazards in industrialising countries. 


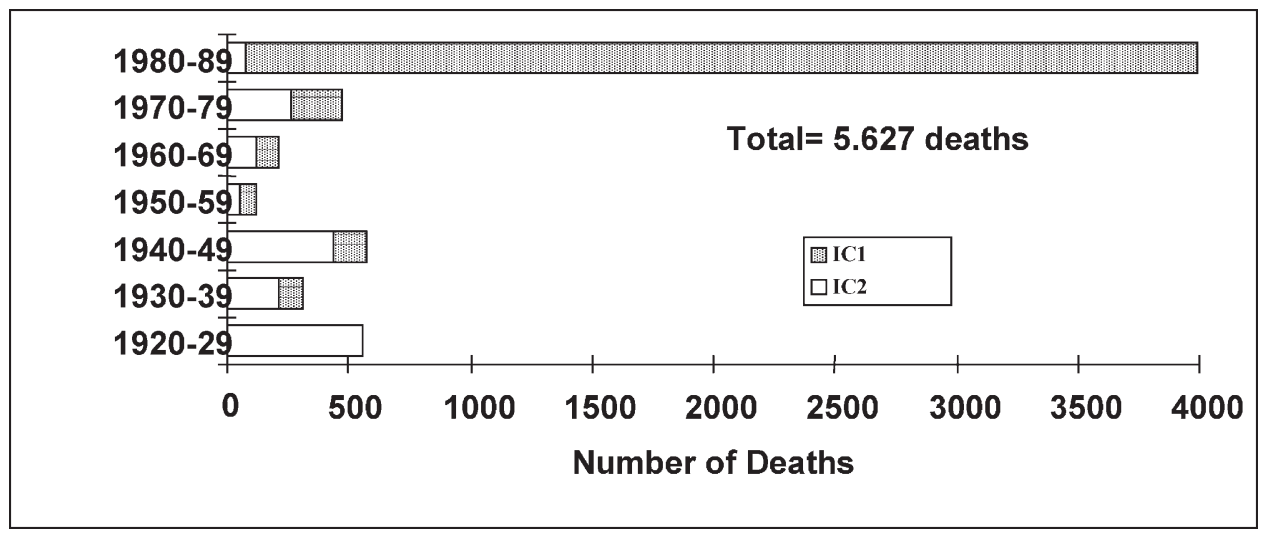

Fig. 4. Number of deaths of major chemical accidents in industrialized and industrializing countries between 1920 E 1989.

Paulo, Brazil and San Juan Ixhuatepec, Mexico). The death figures were respectively 2500 in Bhopal, 508 in Brazil and 550 in Mexico. [25]. These three countries had similar development models in the 1980s. They had high rates of economic growth from the 1960s to the 1980s, but at the cost of developing a huge external debt, of increasing internalization of international corporations and of strong state intervention in the economy. The search for rapid economic growth and accelerated insertion in the global economic system, led to an industrialization model that was further sustained by the absence or weakness of democratic political systems and by deep changes in the structure and organization of society [10,26]. Then, as we can see in Fig. 4, the growing number of victims resulting from major chemical accidents during the 1980s in these countries isn't coincidental, making countries such as Brazil, India and Mexico leaders in deaths per accident, an indicator of severity, as we can see in Table 1.

Table 1

Major chemical accidents with 5 or more deaths between 1945 and $1991^{\text {a }}$

\begin{tabular}{|c|c|c|c|c|c|c|}
\hline Countries & $\begin{array}{l}\text { Accidents } \\
\mathrm{n}\end{array}$ & place & $\begin{array}{l}\text { Deaths } \\
\mathrm{n}\end{array}$ & place & $\begin{array}{l}\text { Deaths/Accidents } \\
\mathrm{n}\end{array}$ & place \\
\hline USA & 144 & 1 & 2.241 & 2 & 15,6 & 8 \\
\hline Japan & 30 & 2 & 526 & 5 & 17,5 & 6 \\
\hline India & 18 & 3 & 4.430 & 1 & 246,1 & 1 \\
\hline Federal Germany & 18 & 3 & 158 & 10 & 8,8 & 10 \\
\hline Mexico & 17 & 4 & 848 & 3 & 49,9 & 3 \\
\hline French & 15 & 5 & 236 & 8 & 15,7 & 7 \\
\hline Italy & 14 & 6 & 260 & 7 & 18,6 & 5 \\
\hline Brazil & 13 & 7 & 815 & 4 & 62,7 & 2 \\
\hline China & 13 & 7 & 454 & 6 & 34,9 & 4 \\
\hline England & 13 & 7 & 170 & 9 & 13,1 & 9 \\
\hline
\end{tabular}

\footnotetext{
a Source: Glickman et al., 1993, [27]
} 
The adoption of this development model brought in its wake a rapid and disordered industrialization and an intense and uncontrolled urbanization process. The large migratory flow from rural and poor areas to the industrial-urban centers was not met by an equivalent number of offers in the job market, nor by the necessary expansion in basic infrastructure that could guarantee to the newcomers minimum conditions in terms of housing, sanitation and health care [28,29]. As a consequence of this process a large number of low-income and impoverished communities had no alternative but to settle in areas adjacent to potentially dangerous chemical plants, thus becoming, for social reasons, highly vulnerable to major chemical accidents.

Moreover, the elite's vested interest in rapid economic growth and industrialization encouraged its members to neglect or totally ignore the need for specific regulations to protect the environment and workers from industrial chemical risks. [30,31] Pressure groups and workers' organizations have been unable to counteract this trend and to press successfully for regulations such as the Seveso Directive in European Communities and the Emergency Planning and Right-to-Know in the USA.

In Brazil, for example, the net result of this process is the absence or weakness of most institutional strategies for prevention and control of industrial accidents and wastes, such as the sites of hazardous facilities and land-use planning, risk analysis, hazardous waste management-including treatment and disposal facilities, obligatory accident notification, emergency planning and the dissemination of information among community members and workers and populations on risks, and strategies for critical situations. This absence or weakness of strategies for prevention and control of industrial hazards seems to be common in other industrializing countries [8].

The legislation regulating the decision-making process for new hazardous installations in industrializing countries is very precarious in practically all of these countries. Hardly any country in Latin America has legislation equivalent to the European "Seveso Directive" or to the North American "Emergency Planning and Right-toKnow". In the cases where legislation exists, the technical and financial deficiencies of the regulatory institutions, added to the lack of efficient pressure groups in political systems that are not fully democratic, strongly limits the enforcement of the law.

In Brazil, the technical difficulties of controlling industrial risks through regulation have been aggravated by the financial crisis that assailed recent governments. This means that many of the existing hazardous plants have been built without effective government control, and safety becomes dependent almost exclusively on the selfregulation of industries in programmes such as responsible care, which are only rudimentary in Brazil. Thus, a decision about the unacceptability of a particular hazardous plant is always delayed until later, being considered only when some catastrophic effects have already occurred. By then, implementing new preventive measures or closing down the industry may no longer be so easily accomplished. The region around the plant will have become economically dependent on its presence, and preventive measures become too expensive to implement once the design and building phases have been completed. The absence of competent technical forums where questions such as the choice of technology, technology transfer and plant design can be discussed and whose recommendations could provide the techni- 
cal elements necessary for optimizing the result of the decision making process, further reduces the possibility that the adopted technology will be the safest one.

The decision-making process on the acceptability of risks is essentially a political process. The formulation of the problem, the right to participate in the decision process, the responsibilities specified by legislation and the ability to influence or constrain the actions of other actors can all strongly influence or even determine the course of judgments. This social process can be understood as a social control of risks and expresses the form in which a society accepts and influences the control of risks created by technological and industrial development. The prevailing interests in society in most cases, and in all its phases, influence the formulation of a technical risk analysis. This happens also in industrialized countries, a fact that can be easily noticed by an examination of technical reports and results on the acceptability of liquefied energy gas terminal [17]. In industrializing countries, the decision-making process is normally restricted to commissions that are closed to representatives of workers' organizations, or of ecological and citizens' groups. In any case these are usually not strong or organized enough to succeed in having their interests met. This obviously limits the effectiveness of the social control of risks and increases the vulnerability of some social groups.

One of the main causes for the increase in the number of major chemical accidents in developing countries is related to the sites of hazardous industries in highly populated or chaotically urbanized areas. As suggested before, in these countries, rapid and disordered industrialization-associated with the process of modernization in the countryside with a view to increasing the export of staples-led also to disordered migration from the countryside to urban centers. Jobless and homeless, these people settled in the peripheries or poorer sectors of the cities. This, combined with local and regional governments' inability to-or lack of interest in-drawing and implementing feasible urban plans, gave rise to a series of slums or poor settlements that shared the space with hazardous industrial sites. The mix of poverty and lack of information are responsible for a typical pattern of major accidents in industrializing countries. In Brazil, two of the most important accidents in terms of deaths (Pojuca in 1983 and Cubatão in 1984) happened when a poor population was trying to store gasoline which had spilled from a train and a pipeline respectively.

It is not a coincidence, therefore, that the victims of the three most serious major chemical accidents in the number of fatalities that happened in the second half of the 20th century-namely in Mexico, Brazil and India, all in 1984-were largely members of these marginalized communities. A comparison between the growing rates of the slum population and those of the population as a whole in the State of São Paulo-Latin America's main industrial metropolis and where the worst major chemical accident in Brazil took place-is revealing: between 1973 and 1987, the total population grew by approximately two-thirds, whereas the population in the slums grew by more than tenfold [32]. The mushrooming of these poor and densely populated areas next to industrial plants is the origin of the social vulnerability of this sector of the population. At the same time, the political weakness of these social groups limits their ability to press for the creation and implementation of new social policies and regulatory practices. 


\section{Vulnerability and prevention in industrialising countries}

The social relations in the workplace play an important role in the origin of industrial accidents at the level of operational prevention [8]. In developing countries the lack of technical qualifications and formal education of the workforce should in theory lead to an extra effort by employers to compensate for the workers' low training levels, especially in the case of technology transfer. This is largely not the case, however, because in these countries, effective labour legislation tends to be non- existent or is easily evaded by employers, and therefore, it becomes easier for them to dismiss workers. The high turnover rates act both as a form of maintaining incomes at low levels and as a way to weaken workers' organizations. And weak workers' organizations mean less pressure for safety and risk control in the workplace. In Bhopal, previous to the accident, the number of blue-collar workers had been reduced from 850 to 642 over a two-year period and qualified plant operators had been replaced by less qualified workers [18]. This dismissal of experienced workers was listed as one of the many causes of the accident. In Brazil, high turnover rates and the replacement of experienced by non-experienced workers are a common fact, in recent years. Also a strained and hierarchical mode of work relations, in making communication between the various work groups more difficult, limits the feedback effect - that is, reduces the possibilities after an accident of effectively implementing technical and organizational measures to avoid future similar accidents.

The degeneration of equipment and the lack of safety precautions in industrializing countries can be associated with the transfer of technology and the firms' lack of sufficient investment, normally due to constant financial crises. This also increases the potential for major chemical accidents such as those in Bhopal, São Paulo and San Juan de Ixuatepec in 1984 [33]. Technical, organizational and economic difficulties discourage the maintenance of adequate operations and systems, and this can affect the safety performance. In Bhopal, the demand for carbaryl pesticides had been lower than expected. This reduced the firm's profits and discouraged the owners from making additional investments in safety measures, or equipment modernization. The closing down of the refrigeration system and the reduction of maintenance operators in Bhopal is an example of the type of economy measures that can lead to a major chemical accident. Institutional weakness contributes to increase the coupling of hazardous production systems in this context.

Another essential factor that must be considered in any attempt to explain the magnitude of major accidents in developing countries is the fact that off-site emergency preparedness plans are often not enforced by law in these countries, and therefore tend either not to exist at all or to be less than adequate. This limits the possibilities for effective mitigation of consequences during an accident, and for remediation afterwards. Since no official report is widely publicised after an accident, the population living in the neighbourhood of the hazardous site-usually densely populated slums as was the case in Bhopal, where density rates were on the range of 25,000 people per square kilometre-react instinctively. This leads to disordered spontaneous evacuation or situations of total panic that aggravate the impact of the 
accident, killing, injuring or in any way affecting the health of a greater number of people.

In Bhopal, it was found that whereas most of the people living in well-built houses escaped the worst effects of the accident, those living under the primitive conditions of the densely populated shanty towns were unable to protect themselves from the gas emitted by the accident. In Brazil, the worst major accident that had ever happened (1984), — with 508 casualties — had its effects made worse by the fact that the surrounding population, in their poverty and ignorance, began to store the spilt gasoline in the hope of being able to sell or use it later, thus creating an inflammable path between their own poorly built houses and the source of the accident and provoking further explosions and fires. By comparing two similar accidents, one in Feyzin (France, 1966) and the other in San Juan Ixhuatepec (Mexico, 1984) it becomes clear how the proximity of densely populated and poorly built houses to hazardous sites can more than double the number of victims of an accident. The explosions in Feyzin in a tank with $6400 \mathrm{~m}^{3}$ of LPG resulted in 17 fatalities and about 80 injuries whereas the accident in San Juan Ixhuatepec, with $12,000 \mathrm{~m}^{3}$ of LPG, killed 500 people and injured 7000. The greatest difference here was the presence of adequate urban planning in Feyzin where the distance of the nearest house to the storage perimeter was in the range of 1000 meters, whilst in Mexico City this distance was only 100 meters. This proximity of houses to chemical plants is, nowadays, a common feature in Brazil.

One of the essential items of emergency planning is the availability of adequate medical facilities. This simply does not happen in industrializing countries. The medical response in Bhopal, for instance, was totally inadequate, in spite of the heroic efforts of the untrained and uninformed local health staff [34]. Generally, in developing countries, the hospitals serving the densely populated and low-income areas that surround chemical plants, due to the so-called diseases of poverty, are permanently overcrowded with patients. They are also understaffed and lacking equipment, drugs and dressings. When a chemical accident occurs, they are simply unable to respond adequately. Here again, social causes such as the lack of public investment in health contribute to an increase in the number of victims of chemical accidents in these countries [35].

\section{Socio-economic rationality regarding vulnerability}

The lack of adequate legislation on industrial chemical risks in developing countries, and the weakness of the existing decision-making processes involving technological risks restrict the social control of risks and allows firms-the risk creators themselves - to do their own regulation with restricted legal enforcement and hardly any government control. Companies' self-regulation in industrializing countries, tends to be based on cost-benefit calculations and a strict economic rationality. Their investments in safety precautions are therefore relatively much lower than the ones in industrialized countries where government regulations and public pressure are much stronger. Another important instrument of prevention is the requirement that 
firms absorb the environmental and occupational costs resulting from industrial hazards. These potential costs to the firms tend to be lower in industrializing countries, thus discouraging investments in safety measures.

A chemical accident is a costly affair involving destroyed equipment and installations, repair expenses, interruption of production, first aid, and medical equipment and treatment, emergency response facilities, decontamination activities, payment of indemnities and possibly pensions to victims, loss of image and market and so on. Here there are two important questions: the actual cost of an accident; and how these costs are distributed among firms - or insurance companies_-and society as a whole. In a strict economic analysis of safety investments the optimum value is the one which maximizes the relationship between investments and possible costs. The optimum economic safety standard leaves a residual risk that may be economically acceptable. This does not mean, however, that this risk is morally acceptable.

There are some indications that the costs of major accidents, particularly its human costs, are not as expensive in industrializing as they are in industrialized countries. The initial compensation payment requested by the Indian authorities from the Union Carbide was 3 billion dollars, but, in the end, they were satisfied with 470 million. Relatives of the fatal victims received between $\$ 4500$ and $\$ 12,500$; invalids between $\$ 1000$ and $\$ 8000$ and a provisional pension of less than 10 dollars a month was offered to the victims by the Government. If a similar accident had occurred in an industrialized country, costs would have been much higher than these. [36].

Economic values are also expressions of ethics and social and economic policies. The Post-Normal Science approach can help us to understand this not only with respect to complex environmental problems but also with more general environmental and health issues. With this approach, what might seem 'simple' hazards are revealed as much more complex, as they incorporate social vulnerability. When the human costs of industrial accidents are seen as 'normal' lost lives in conformity with the market economic rationality, there is no barrier to the pressures for the lowering of technical preventive measures in industrializing countries in a globalised and unequal world.

\section{Conclusion: possibilities of changes in industrial hazards in a globalised world}

The contemporary organization of the global economy, with its international division of labour, and of benefits and risks, has been contributing to the vulnerability of industrializing countries in facing industrial hazards, along with other environmental risks. Reversing the vulnerability of industrializing countries involves initiatives of varying nature and degrees of complexity, both at national and international levels.

There are some important consequences and challenges of integrated approaches in trying to reduce vulnerability in industrializing countries. These strategies aim to prevent several vicious circles, which sustain the externalization of social and environmental costs of industrial hazards from the enterprises that create them. The points below are suggestions from the point of view of integrated assessment to research teams and institutional policies in industrializing countries. 
1. To organize different levels of possible interventions in a systemic way, linking global and local strategies, ranging from from structural policies and institutional practices to actions of local social actors-e.g. regulatory agencies, industries, workers, populations in hazardous areas;

2. A better understanding of the limits and uncertainties of local and 'normal' scientific diagnosis and proposals;

3. Empowerment of vulnerable groups as an important strategy for research teams and institutions in a dialectic way of changing society and science together;

4. To contextualize the understanding of industrial hazards within the needs and priorities of vulnerable populations, developing new forms of dialogues and risk communication with poor and less formally educated people, creating possibilities of reciprocal exchanges between lay and scientific knowledge.

But there still remains an important question in terms of changes in the development model of industrializing countries. Is it possible to combine internalization and competitiveness in industrializing countries in a global market with equity and sustainability? We believe that this could be possible if the society incorporates not only the economic approach in the scientific and politic debate about environment hazards, but also employs a new integrated approach, such as we can see in the perspective of Ecological Economics. This would repair the classic division of scientific knowledge between 'hard' natural and 'soft' human sciences , enhancing “...our understand of both predominantly natural and predominantly cultural ('reflexive') systems through their poetry", and centred in the concept of Quality for the synthesis of systems thinking [37]. In this, the essential element is comprehension of the limits of normal scientific language in grasping the qualitative and ethical aspects of life.

A global approach reveals that social vulnerability within hazardous sites in industrializing countries will not be reduced without the devising and implementation of major social policies, both at local and global levels. In those countries, a large part of the obstacles to the regulation of industrial risks can be overcome only by changing the socio-political structures that amplify the frequency and effects of chemical and other industrial risks. For this to happen, however, we must strive for two things. First, at the international level, there must be a deep transformation of the social and environmental inequalities that characterize the present global economic system is needed. Second, at the national level, there must be a more intensive participation of the involved actors - and this means an effective redistribution of wealth and the consolidation of civil rights for all - to guarantee the modernization and enforcement of appropriate legislation and the elimination of other obstacles.

In order to reduce the vulnerability of industrializing countries to technological hazards and achieve a global ecological change, it should be necessary for all of spaceship Earth's passengers to have the right to discuss and participate together in the decision making process about industrial hazards, achieving similar sets of basic protection measures. The fact that there are different classes of passengers in this ship may jeopardize the development of a common alternative solution for the present impasse that is simultaneously social, political, economic, cultural, scientific and ethical. The proposal of the Post-Normal Science approach can contribute to inspire 
institutional changes towards solving environmental problems. Thus unfortunate concepts such "Third" and "First" worlds should be forgotten if we genuinely believe that both industrial hazards and future ecological disasters must be prevented.

\section{Acknowledgements}

We acknowledge the discussions with Silvio Funtowicz, Bruna De Marchi and Claudia Natenzon.

\section{References}

[1] T. Horlick-Jones, Patterns of risk and patterns of vulnerability, in: A. Amendola, B. de Marchi (Eds.), Workshop on Emergency Management, Comission of the European Communities-Joint Research Centre-Institute for Systems Engineering and Informatics, Italy, 1993, pp. 33-42.

[2] P. Winchester, Power, Choice and Vulnerability: A Case Study in Disaster Management in South India, 1977-1988, James \& James, London, 1992.

[3] R. Garcia, Interdisciplinaridade y sistemas complejos, in: E. Leff, R. Garcia, P. Gutman, V. Toledo, H. Vessuri, R. Fernández, R. Brañes (Eds.), Ciencias Sociales y formación ambiental, Editorial Gedisa, Barcelona, 1994, pp. 85-124.

[4] S. Funtowicz, J. Ravetz, Uncertainty and Quality in Science for Policy, Kluwer Publishers, Dordrecht, 1990.

[5] S. Funtowicz, J. Ravetz, Epistemologia Politica: Ciencia con la gente, Centro Edit. Am. Latina, Buenos Aires, 1993.

[6] S. Funtowicz, J. Ravetz, Emerging complex systems, Futures 26 (1994) 6.

[7] T.S. Kuhn, A Eestrutura das Revoluções Científicas, Ed Perspectiva, São Paulo, 1987.

[8] M. Porto, C. Freitas, Major chemical accidents in industrializing countries: the socio-political amplification of risk, Risk Analysis 16 (1996) 19-29.

[9] I. Wallerstein, The Capitalist World Economy, Cambridge University Press, New York, 1979.

[10] B. Becker, C. Egler, Brasil: Uma Nova Potencial Regional na Economia Mundo, Bertrand, São Paulo, 1993.

[11] United Nations Development Program, Human Development Report. Geneve, 1998.

[12] H.S. Brown, J.J. Himelberger, A.L. White, Development of environment interactions in the export of hazardous technologies - a comparative study of three multinational affiliates in developing countries, Technological Forecasting and Social Change 43 (1993) 125-155.

[13] C. Perrow, Normal Accidents: Living with High-Risks Technologies, Basic Books, New York, 1984.

[14] B. Wynne, Risk assessment of technological system—dimensions of uncertainty, in: B. Wynne (Ed.), Risk Management and Hazardous Waste-Implementation and Dialectics of Credibility, SpringerVerlag, Berlin, 1987, pp. 356-398.

[15] S. Funtowicz, J. Ravetz, A framework for the analysis of emergencies, in: A. Amendola, B. de Marchi (Eds.), Workshop on Emergency Management, Comission of the European CommunitiesJoint Research Centre-Institute for Systems Engineering and Informatics, Italy, 1993, pp. 33-42.

[16] H. Otway, Regulation and risk analysis, in: H. Otway, M. Peltu (Eds.), Regulation Industrial RisksScience, Hazards and Public Protection, Butterworths, London, 1985, pp. 01-19.

[17] H.C. Kunreuther, J. Linnerooth, Risk analysis and decision processes-the siting of liquefied energy gas facilities in four countries, Springer-Verlag, Berlin, 1983.

[18] B. Castleman, V. Navarro, International mobility of hazardous products, industries and wastes, Ann. Rev. of Public Health 8 (1987) 01-09.

[19] R. Bullard, Overcoming racism in environmental decision, Environment 36 (1994) 4. 
[20] R.E. Kasperson, et al. The social amplification of risk: a conceptual framework, Risk Analysis 8 (1988) 177-187.

[21] C. Freitas, M. Porto, J. Machado (Eds.), Acidentes Industriais Ampliados: Desafios e Perspectivas, Ed. FIOCRUZ, Rio de Janeiro, 1999.

[22] Economic European Communities, Council Directive of 24 June 1982 on the Major Hazards of Certain Industrial Activities-85/501/E.E.C. (Seveso Directive).

[23] P. Carson, C.J. Munford, Reporting and analysis of industrial incidents-1981-1986, Industry and Environment 11 (1988) 23-29.

[24] Organization for Economic Co-Operation and Development, Health Aspects of Chemical Accidents-Guidance on Chemical Accident Awareness, Preparedness and Response for Health Professionals and Emergency Responders, Organization for Economic Co-Operation and Development, Paris, 1994.

[25] World Health Organization, WHO Commission on Health and Environment-Report of the Panel on Industry, World Health Organization, Geneva, 1992.

[26] F.H. Cardoso, E. Faleto, Dependência e Desenvolvimento na América Latina-Ensaio de Interpretação Sociológica, Zahar, Rio de Janeiro, 1979.

[27] T.S. Glickman, et al. Fatal Hazardous Materials Accidents in Industry-Domestic and Foreign Experience from 1945-1991, Center for Risk Management, Washington, 1993.

[28] D.L. Botz, Manufacturing poverty: the maquiladorization of Mexico, International Journal of Health Services 24 (1994) 403-408.

[29] American Public Health Association, The public health implications of the Bhopal disaster-report to the program development board, American Public Health Association-Bophal Working Group, American Journal of Public Health, 77 (1987) 230-236.

[30] M. Merceir, Chemical safety as a major challenge for developing countries, Biomedical and Environmental Sciences 3 (1992) 211-216.

[31] S. Jasanoff, The Bhopal disaster and the right to know, Social Science and Medicine 27 (1988) 1113-1123.

[32] L.C. Ribeiro, L.C. Lago, Crise e Mudanças nas Metrópoles Brasileiras: A Periferização da Questão, in: M.C. Leal (Ed.), Saúde, Ambiente e Desenvolvimento-Uma Análise Interdisciplinar, volume I, HUCITEC-ABRASCO, Rio de Janeiro, 1992, pp. 153-180.

[33] J. Jeyaratan, The transfer of hazardous industries, Journal of the Society of Occupational Medicine 40 (1990) 123-126.

[34] J.P. Koplan, H. Falk, G. Garet, Public health lessons from the Bhophal chemical disaster, Journal of American Health Association 264 (1990) 2795-2796.

[35] E.A. Vasconcellos, O Atendimento Médico de Emergência nos Acidentes Químicos Ampliados. Dissertation M.P.H. Escola Nacional de Saúde Pública da Fundação Oswaldo Cruz, Rio de Janeiro, 1995.

[36] Badische Zeitung, Noch immer sterben die Meschen in Bhopal (13.08.1992), p. 13, (in German).

[37] S. Funtowicz, J. Ravetz, The poetry of thermodynamics: energy, entropy/exergy and quality, Futures 29 (9) (1997) 791-810. 